\title{
BioFeedback
}

\section{[Letter to the editor] \\ Commercial Cdk1 antibodies recognize the centrosomal protein Cep152}

The centrosome is a highly organized cellular organelle that functions as the main microtubule-organizing center (MTOC) of animal cells and is crucial for fundamental cellular processes (1). Proteomic analyses have revealed a network of interactions among centrosomal proteins $(2,3)$. We report here that two widely used commercial mouse monoclonal antibodies against the master cell cycle regulator Cdk1 (sc-54, Santa Cruz Biotechnology, Dallas, TX; ab18, Abcam, Cambridge, United Kingdom) cross-react with the centrosomal protein Cep152. Our results provide an explanation for previous conflicting reports on the localization of Cdk1 and also question the reported centrosomal recruitment of C $\mathrm{dk} 1$ by Cep63.

Recently, we reported the existence of a Cep57-Cep63-Cep152 centrosomal complex, in which Cep63 and Cep152 exhibit a strong mutual dependency for their localization at centrosomes (4). Given that Cep63 has been reported to be required for the presence of the master cell cycle regulator Cdk1 at centrosomes $(5,6)$, we expected that centrosomal Cdk1 localization should also depend on Cep152 function. To test this hypothesis, we carried out pull-down experiments, performing Western blot analysis of the fusion proteins purified from HEK293 cells expressing SNAP-Cep152, SNAP-Cep63, SNAP-Cep57, or Cdk1-SNAP. SNAP-tag is a $20 \mathrm{kD}$ protein tag that can be covalently labeled (e.g. with fluorescent dyes), thus permitting detection of the fused proteins in vitro and in vivo (7-9). We probed the membrane with the mouse monoclonal anti-Cdk1 antibody sc-54 that was utilized in the study reporting the dependency of centrosomal Cdk 1 on Cep63 function (5). This antibody has also been used in over 200 published studies (www.scbt.com/datasheet-54cdc2-p34-17-antibody.html).

Surprisingly, we found that sc-54 recognized not only $\mathrm{Cdk} 1$, but also Cep152 (Figure 1). To clarify this issue, we purchased four other commercial anti-Cdk 1 antibodies that were raised against other Cdk1 immunogens and repeated the Western blot analysis (Table 1, Figure 1, Supplementary Figure S3B). We found that the mouse monoclonal anti-Cdk1 antibody ab18 similarly cross-reacted with Cep152, whereas the rabbit polyclonal HPA003387 antibody or mouse monoclonal antibodies ab8040 and 610038 did not (Figure 1).

The observed cross-reactivity of some anti-Cdk1 antibodies does not pose a significant problem in simple Western blotting experiments because of the size difference between Cdk1 and Cep152, but it could potentially cause misleading results in immunofluorescence and pull-down experiments. We therefore set out to perform immunofluorescence with all five antibodies to determine whether the cross-reactivity observed by Western blot also applies to this experi- mental approach, which was previously used to report the reliance of centrosomal Cdk1 on Cep63 function (5). We fixed control cells and cells depleted of Cdk1 or Cep152, using two distinct siRNAs/siLNAs corresponding to each protein. The resulting samples were then stained with anti-Cdk 1 antibodies (one antibody per sample), along with anti- $\gamma$ tubulin antibodies to mark centrosomes. In each case, we quantified the resulting centrosomal signal intensity in cells in late G2/early mitosis relative to the $\gamma$-tubulin signal; the outcome of these experiments is summarized in Table 1 , and more detailed results are listed in Supplementary Table S1. First, we found that both sc-54 and ab18 antibodies labeled centrosomes in control conditions throughout the cell cycle, as reported previously for sc-54 (10). These results contrast with earlier studies using PSTAIR and CTR 453 antibodies, which led to the conclusion that $\mathrm{Cdk} 1$ localizes to centrosomes solely in late G2/early mitosis (11). In accordance with the latter result, we found that in control conditions the HPA003387, ab8040, and 610038 antibodies indeed significantly labeled centrosomes solely during late G2/early mitosis (Supplementary Figure S1A). Second, we found that the centrosomal signal of the sc-54 and ab18 antibodies decreased substantially upon depletion of Cep152 but not upon depletion of Cdk1 (Table 1, Supplementary Table S1, Supplementary Figure S1B). Conversely, we found

Table 1. Properties of tested Cdk1 antibodies

\begin{tabular}{|c|c|c|c|c|c|c|c|}
\hline \multirow{2}{*}{ Cdk1 Antibody (Company) } & \multicolumn{2}{c}{ Species and type } & \multicolumn{2}{c}{ Signal intensity after depletion of: } & \multicolumn{2}{c|}{ Present solely in late G2/mitosis } & Recognizes SNAP-Cep152 on WB \\
\hline sc-54 (Santa-Cruz) & Mouse monoclonal & High & Low & Low & NO & YES \\
\hline ab18 (Abcam) & Mouse monoclonal & High & Low & ND & NO & YES \\
\hline HPA003387* (Sigma-Aldrich) & Rabbit polyclonal & Low & High & ND & YES & NO \\
\hline ab8040* (Abcam) & Mouse monoclonal & Low & High & High & YES & NO \\
\hline $610038^{*}$ (BD Biosciences) & Mouse monoclonal & Low & High & High & YES & NO \\
\hline
\end{tabular}

A minimum of 13 cells (26 centrosomes) were analyzed in each case. * indicates that the centrosomal signal was measured only in cells in late G2/ early mitosis. High signal intensity corresponds to normalized median values $>0.5$; low signal intensity corresponds to normalized median values $<0.5$ (see Supplementary Table S1). ND: not determined. 


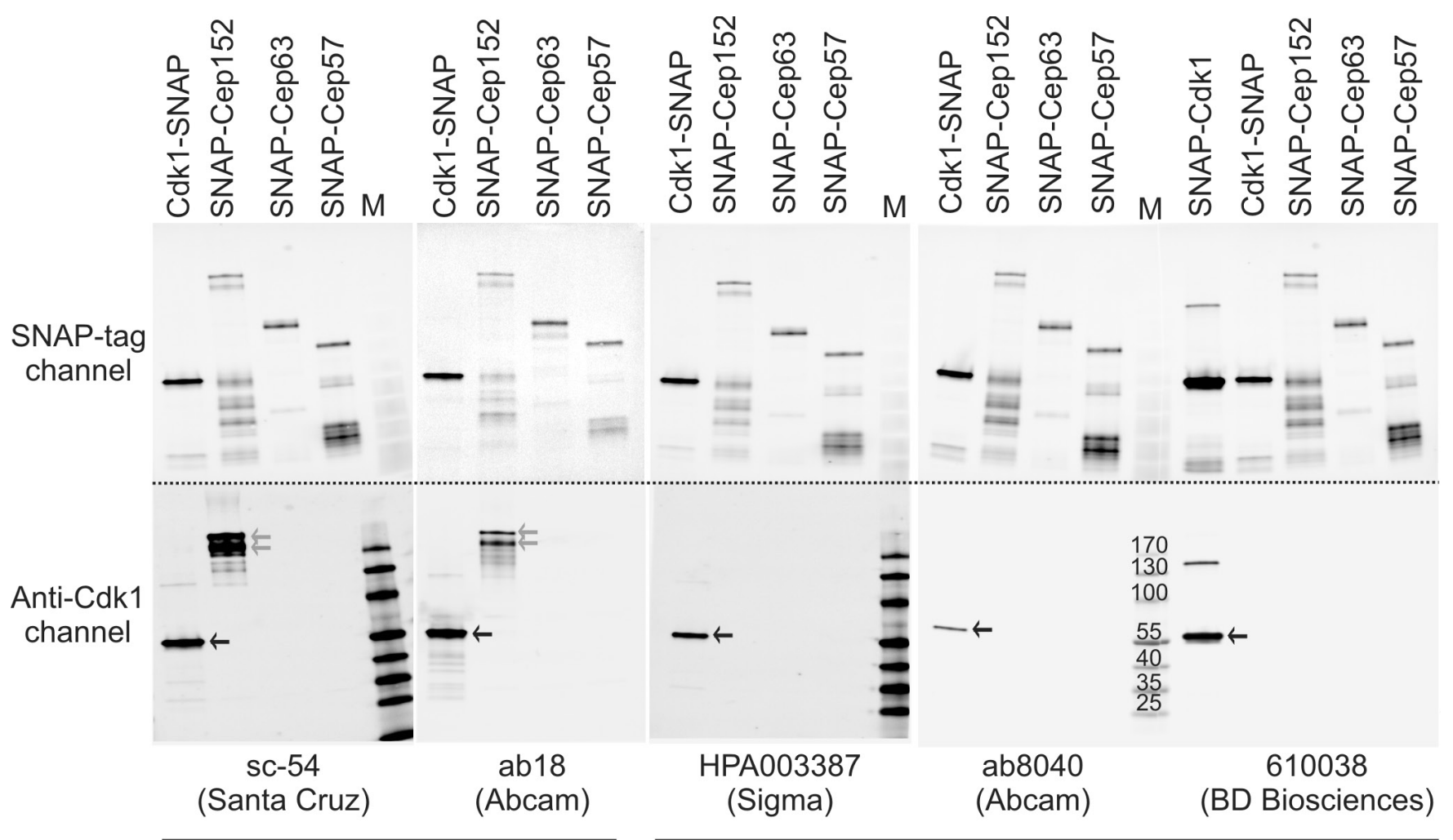

Cross-reactive with Cep152

No cross-reactivity with Cep152

Figure 1. Characterization of Cdk1 antibodies using Western blotting. Western blots with five different Cdk1 antibodies are presented to illustrate the reactivity of antibodies to SNAP-tag fusion proteins purified from HEK 293 cells. The upper row shows Western blot membranes scanned for SNAP-tag fluorescence labeled with SNAP-Surface 488 substrate. The lower row shows Western blot membranes scanned for Cdk1 antibody fluorescence detected with an appropriate secondary antibody labeled with Alexa647. Note that the 610038 (BD Biosciences) antibody recognizes only the free Cdk1 C-terminus and thus detects SNAP-Cdk1 but not Cdk1-SNAP. Black arrows indicate SNAP-tagged Cdk1. Gray arrows indicate SNAP-Cep152. M indicates the PageRuler prestained protein ladder; numbers above PageRuler bands indicate molecular weight in kDa.

little or no change in the centrosomal signals for the HPA003387, ab8040, or 610038 antibodies upon depletion of Cep152, but a substantial decrease was observed upon depletion of Cdk1 (Table 1, Supplementary Table S1). Analogous results were obtained when Cep63 was depleted by RNA interference (Supplementary Figure $\mathrm{S} 1 \mathrm{~B}$ ), as expected given that Cep63 and Cep152 are mutually dependent for their centrosomal localization (4).

Next, we conducted confocal imaging of U2OS cells to precisely localize the centrosomal signal of anti-Cdk1 antibodies relative to $\mathrm{C}-\mathrm{Nap} 1$, which marks the proximal end of the centriole, or Cep152, which is present in a ring just above the proximal end $(6,12-15)$. The confocal microscopy experiments established that both sc-54 and 610038 antibodies labeled the proximal end of the centriole (Supplementary Figure S2 A and B). Interestingly, however, we found that the distribution of the sc-54 signal was different from that of the 610038 signal, with the former matching the localization of the Cep152 ring perfectly (Supplementary Figure S2C and D). To confirm this, we generated a U2OS cell line expressing a Cdk1-SNAP fusion protein. Immunofluorescence experiments showed that the Cdk1-SNAP signal resembled the staining obtained with the 610038 antibody and not the sc-54 antibody (Supplementary Figure S2E and F). To investigate whether the distribution of Cep152 and Cdk1 at centrosomes may differ, we performed STED (stimulated emission depletion) microscopy. In accordance with previous observations (4), super-resolution microscopy showed that Cep152 was present at the proximal end of the parental centriole in a ring-like structure $367 \mathrm{~nm}$ in diameter on average (Supplementary Figure S3A). By contrast, we found that the 610038 antibody signal was positioned in a much more diffuse manner, most likely in the pericentriolar material (PCM), and could not be resolved as a ring by
STED microscopy (Supplementary Figure S3A).

Finally, we found that there may be a simple explanation for the observed cross-reactivity between Cdk1 and Cep152 using the sc-54 and the ab18 antibodies. Indeed, these antibodies were raised against partially overlapping immunogens of Cdk1, which exhibit some degree of similarity with the amino acid sequence of Cep152 (Supplementary Figure S3B and C).

In conclusion, our observations indicate that both sc-54 and ab18 antibodies recognize not only Cdk1 but also Cep152 in Western blot and immunofluorescence assays. By contrast, HPA003387, ab8040, and 610038 appear to recognize Cdk1 specifically, and demonstrate that $\mathrm{Cdk} 1$ localizes most likely in the PCM of centrosomes in a cell-cycle dependent manner. Our work also serves as a reminder that numerous independent control experiments are needed to verify antibody specificity in immunofluorescence and other antibody-based experiments (16). 


\section{Acknowledgments}

The authors thank Fernando Romero Balestra, Meritxell Orpinell and Rūta Gerasimaite for their useful comments on the manuscript.

\section{Competing interests}

The authors declare no competing interests.

\section{References}

- Superior biotin-free nanopolymer detection system for the lowest background

- All-in-one multiplex kits preserve time and precious tissue samples

- Broadest palette of HighDef ${ }^{\mathrm{TM}}$ chromogens including unique HighDef ${ }^{\mathrm{TM}}$ Yellow

- Over $1000 \mathrm{IHC}$ validated antibodies for worry-free detection

\section{www.enzolifesciences.com/IHC}

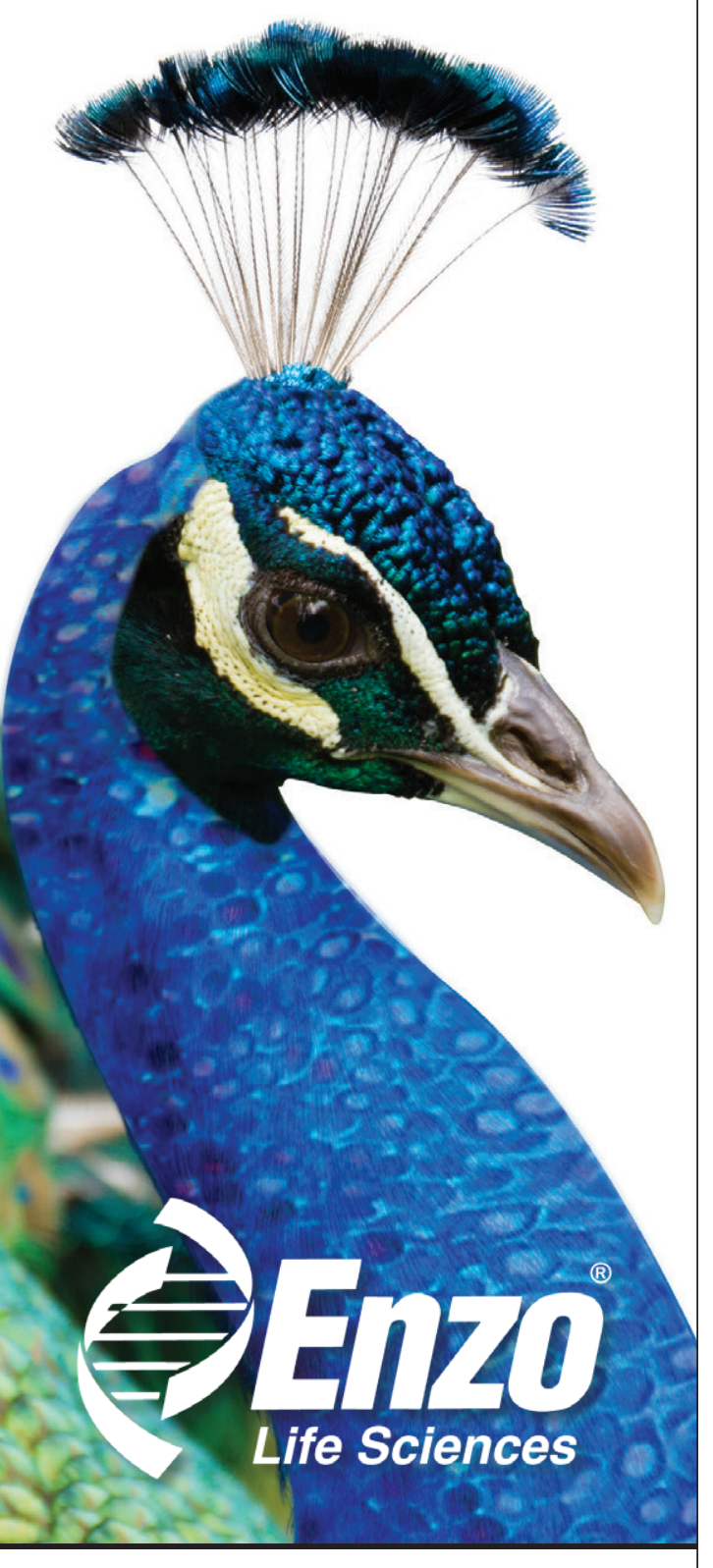

. Gönczy, P. 2012. Towards a molecular architecture of centriole assembly. Nat. Rev. Mol. Cell Biol. 13:425-435.

2. Andersen, J.S., C.J. Wilkinson, T. Mayor, P. Mortensen, E.A. Nigg, and M. Mann. 2003. Proteomic characterization of the human centrosome by protein correlation profiling. Nature 426:570-574.

3. Jakobsen, L., K. Vanselow, M. Skogs, Y. Toyoda, E. Lundberg, I. Poser, L.G. Falkenby, M. Bennetzen, et al. 2011. Novel asymmetrically localizing components of human centrosomes identified by complementary proteomics methods. EMBO J. 30:1520-1535.

4. Lukinavičius, G., D. Lavogina, M. Orpinell, K. Umezawa, L. Reymond, N. Garin, P. Gönczy, and K. Johnsson. 2013. Selective chemical crosslinking reveals a cep57-cep63-cep152 centrosomal complex. Curr. Biol. 23:265-270.

5. Löffler, H., A. Fechter, M. Matuszewska, R. Saffrich, M. Mistrik, J. Marhold, C. Hornung, F. Westermann, et al. 2011. Cep63 recruits Cdk1 to the centrosome: implications for regulation of mitotic entry, centrosome amplification, and genome maintenance. Cancer Res. 71:2129-2139.

6. Sir, J.H., A.R. Barr, A.K. Nicholas, O.P. Carvalho, M. Khurshid, A. Sossick, S. Reichelt, C. D'Santos, et al. 2011. A primary microcephaly protein complex forms a ring around parental centrioles. Nat Genet.

7. Keppler, A., S. Gendreizig, T. Gronemeyer, H. Pick, H. Vogel, and K. Johnsson. 2003. A general method for the covalent labeling of fusion proteins with small molecules in vivo. Nat. Biotechnol. 21:86-89.

8. Mollwitz, B., E. Brunk, S. Schmitt, F. Pojer, M. Bannwarth, M. Schiltz, U. Rothlisberger, and K. Johnsson. 2012. Directed evolution of the suicide protein $\mathrm{O}(6)$-alkylguanine-DNA alkyltransferase for increased reactivity results in an alkylated protein with exceptional stability. Biochemistry 51:986-994.

9. Bojkowska, K., F. Santoni de Sio, I. Barde, S. Offner, S. Verp, C. Heinis, K. Johnsson, and D. Trono. 2011. Measuring in vivo protein half-life. Chem. Biol. 18:805-815.

10.Pockwinse, S.M., G. Krockmalnic, S.J. Doxsey, J. Nickerson, J.B. Lian, A.J. van Wijnen, J.L. Stein, G.S. Stein, and S. Penman. 1997. Cell cycle independent interaction of CDC2 with the centrosome, which is associated with the nuclear matrixintermediate filament scaffold. Proc. Natl. Acad. Sci. USA 94:3022-3027.

11.Bailly, E., M. Doree, P. Nurse, and $M$. Bornens. 1989. p34cdc2 is located in both nucleus and cytoplasm; part is centro- somally associated at $\mathrm{G} 2 / \mathrm{M}$ and enters vesicles at anaphase. EMBO J. 8:3985-3995.

12.Conroy, P.C., C. Saladino, T.J. Dantas, P. Lalor, P. Dockery, and C.G. Morrison. 2012. C-NAP1 and rootletin restrain DNA damage-induced centriole splitting and facilitate ciliogenesis. Cell Cycle 11:37693778.

13.Fry, A.M., T. Mayor, P. Meraldi, Y.D. Stierhof, K. Tanaka, and E.A. Nigg. 1998. C-Nap1, a novel centrosomal coiled-coil protein and candidate substrate of the cell cycle-regulated protein kinase Nek2. J. Cell Biol. 141:1563-1574.

14. Mayor, T., Y.D. Stierhof, K. Tanaka, A.M. Fry, and E.A. Nigg. 2000. The centrosomal protein C-Nap1 is required for cell cycleregulated centrosome cohesion. J. Cell Biol. 151:837-846.

15. Dzhindzhev, N.S., Q.D. Yu, K. Weiskopf, G. Tzolovsky, I. Cunha-Ferreira, M. Riparbelli, A. Rodrigues-Martins, M. Bettencourt-Dias, et al. 2010. Asterless is a scaffold for the onset of centriole assembly. Nature 467:714-718.

16.Stadler, C., E. Rexhepaj, V.R. Singan, R.F. Murphy, R. Pepperkok, M. Uhlen, J.C. Simpson, and E. Lundberg. 2013 Immunofluorescence and fluorescentprotein tagging show high correlation for protein localization in mammalian cells. Nat Methods. 10: 315-323.

Received 26 June 2013; accepted 29 July 2013.

Gražvydas Lukinavičius ${ }^{1}$,Darja Lavogina ${ }^{1}$, Pierre Gönczy, and Kai Johnsson ${ }^{1}$

${ }^{1}$ Ecole Polytechnique Fédérale de Lausanne (EPFL), Institute of Chemical Sciences and Engineering (ISIC), Lausanne, Switzerland and ${ }^{2}$ Ecole Polytechnique Fédérale de Lausanne (EPFL), School of Life Sciences, Swiss Institute for Experimental Cancer Research (ISREC); Lausanne, Switzerland.

BioTechniques 55:111-114 (September 2013) doi $10.2144 / 000114074$

Address correspondence to Kai Johnsson, Ecole Polytechnique Fédérale de Lausanne, Institut des sciences et ingénierie chimiques Lausanne, Switzerland, E-mail: kai.johnsson@epfl.ch; or to Pierre Gönczy, Ecole Polytechnique Fédérale de Lausanne, Swiss Institute for Experimental Cancer Research, Lausanne, Switzerland, E-mail: pierre.gonczy@epfl.ch.

Supplementary material for this article is available at www.BioTechniques.com/article/114074.

To purchase reprints of this article, contact: biotechniques@fosterprinting.com 